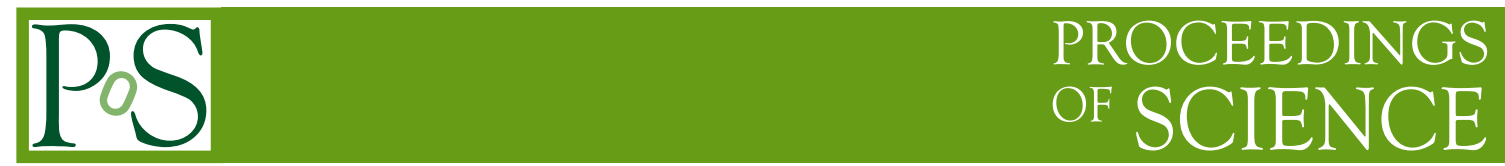

\title{
Development of readout electronics for MPPC
}

\section{Takeshi Murakami*}

IPNS, High Energy Accelerator Research Organization (KEK), Japan

E-mail: takeshi@post.kek.jp

\section{Shinichi Gomi}

Department of Physics, Kyoto University, Japan

We report on the development of read out electronics for Multi-Pixel Photon Counter (MPPC). Using Trip-t frontend chip, we have developed a system that can read out and test 32 MPPC's at once. This system will be highly useful for characterization of a large number of MPPC's.

International Workshop on new Photon-Detectors

June 27-29 2007

Kobe, Japan

\footnotetext{
* Speaker.
} 


\section{Introduction}

We have developed a readout system for Multi-Pixel Photon Counter (MPPC) utilizing Trip$\mathrm{t}^{1}$ ASIC chip developed at Fermilab [1]. Trip-t has 32 input channels and charge, timing and discriminator outputs. Charge and timing information is stored in pipeline and serially read out. Using this system, 32 MPPC's can be read out at once. Design and performance of this system are reported.

\section{Design of the readout system}

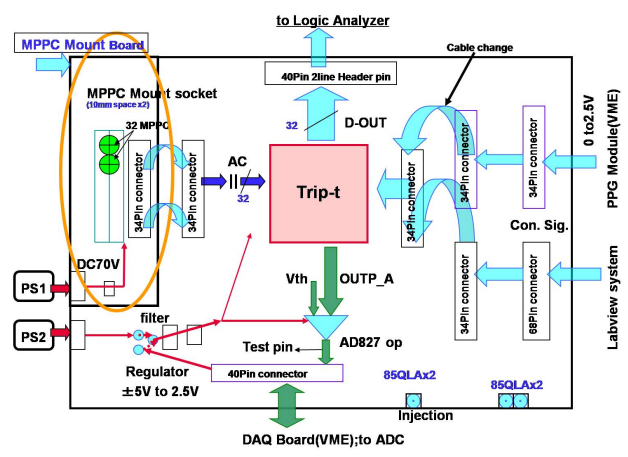

Figure 1: Block diagram of KEK Trip-t board.

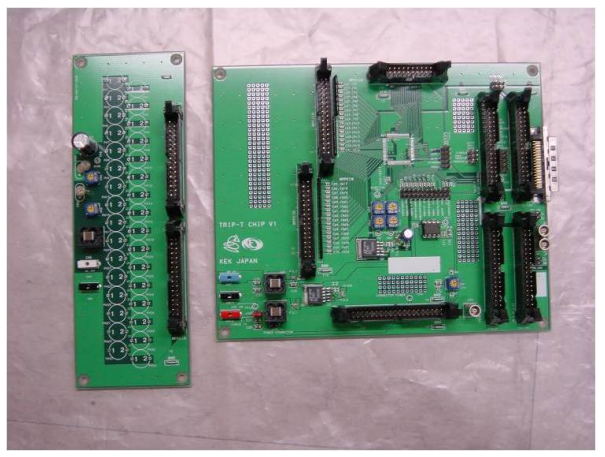

Figure 2: Picture of KEK Trip-t frontend board and MPPC mounting adapter card.

Figure 1 shows the block diagram of the developed frontend board. The number of input channels for this board is 33, one for the gain calibration of Trip-t and 32 for MPPC signals. Control signals are sent from an external pattern generator. We designed the board to be compatible with both a VME PPG module and a digital waveform generator controlled with a LabVIEW program. Figure 2 shows a picture of the developed frontend board together with an MPPC mount card.

The charge information from Trip-t is sent to a VME board equipped with ADC and digitized. This board was originally developed for readout of multi-anode PMT signal in K2K experiment [2]. Discriminator signal can be read with a logic analyzer.

\section{Performance}

First, we test the performance of the system by injecting charge to Trip-t. We measure the dynamic range with three different internal gain settings of Trip-t and confirm the Trip-t is operated as expected. Cross-talk between channels is measured to be around $0.4 \%$.

Then, an MPPC is connected to the readout system. Figure 3 shows the charge output (AOUT) from Trip-t when a channel (channel 10) is connected to an MPPC. A weak light from LED is injected to the MPPC. Clear separation is seen between the output levels corresponding to pedestal and one, two and three photoelectron signal. The ADC distribution also shows clear separation of p.e. peaks as seen in Fig. 4

\footnotetext{
${ }^{1}$ Trip-t is the abbreviation of Trigger and pipeline with timing.
} 


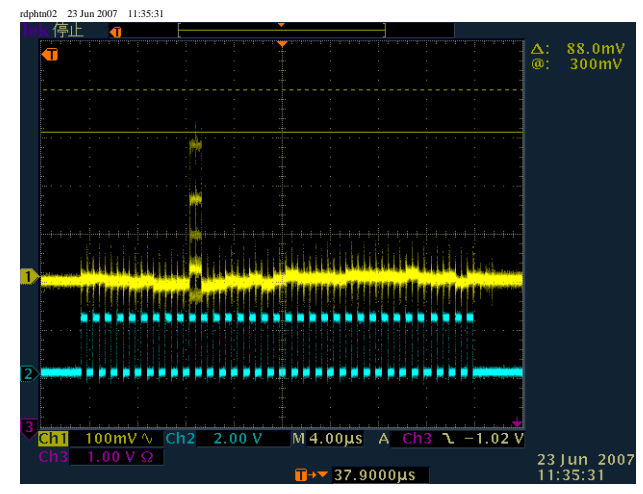

Figure 3: Charge output from

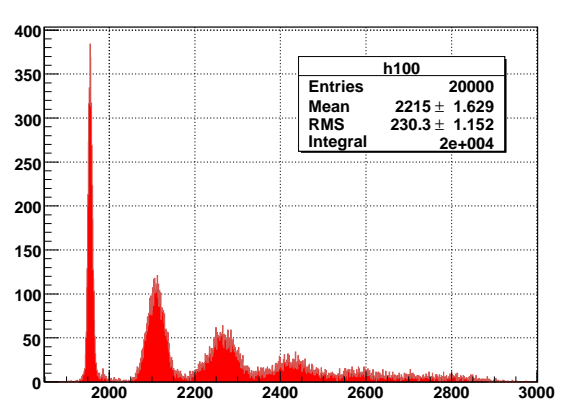

Figure 4: ADC distribution of signal from an MPPC.

Finally, we successfully read out 32 MPPCs at once using this system. So far, 300 MPPC devices are measured using this system as reported in [3]. The system developed in this work is proved to be highly useful for the test of MPPC basic parameters at mass production.

\section{Summary}

A readout system of MPPC is developed utilizing Trip-t frontend ASIC. Using the developed system, 32 MPPC's are read out at once. This system will be useful for characterization of MPPC when a large number of devices are produced.

This work was done as a part of activity in KEK Detector Technology Project, photon sensor group.

\section{References}

[1] J. Estrada, et al., MCM II and the Trip Chip, D0 Note 4009 (2002).

[2] M. Yoshida et al., IEEE Trans. Nucl. Sci. 51, 3043 (2004).

[3] S. Gomi, "R\&D of MPPC for T2K experiment", in these proceedings. 